\title{
The influence of sulfate availability on rheology of fresh cement paste
}

https://doi.org/10.1515/arh-2020-0106

Received Jul 02, 2019; accepted Jun 20, 2020

\begin{abstract}
Natural gypsum can degenerate into hemihydrate during cement clinker grinding which changes the physical and chemical properties of cement hydration, affecting therefore the fresh and hardened properties of cement based materials. Cement systems containing a constant total amount of calcium sulfate $(4 \%)$ with relative proportions of hemihydrate and natural gypsum were considered. Rheological measurements were executed on an Anton Paar MCR51 rheometer to evaluate the flow properties of cement pastes. Results show that, the yield stress and the plastic viscosity of cement pastes were affected when the degeneration of natural gypsum exceeded $50 \%$. Above this concentration, the yield stress remarkably increased and a variation in plastic viscosity of about $50 \%$ was observed. Using TG-DSC techniques, it was shown that, the amount of formed ettringite could not explain these rheological changes. However, centrifugational packing and SEM-SE measurements confirmed that, more than the amount of ettringite precipitated, ettringite morphology plays a major role in controlling the yield stress and plastic viscosity of fresh cement pastes.
\end{abstract}

Keywords: Natural gypsum, hemihydrate, ettringite, rheology and cement paste

\section{Introduction}

In ordinary Portland cement manufacturing, clinker is ground together with calcium sulfate as a source of sulfate to prevent premature and detrimental precipitation of some calcium aluminate phases during the early stage of cement hydration. Subsequently, the aluminate reactiv-

\footnotetext{
*Corresponding Author: Rainer Haldenwang: Department of Civil Engineering, Cape Peninsula University of Technology, PO Box 652 Cape Town 8000, South Africa; Email: haldenwangr@cput.ac.za Willy Mbasha, Irina Masalova: Department of Civil Engineering, Cape Peninsula University of Technology, PO Box 652 Cape Town 8000, South Africa
}

ity is regulated through preferential precipitation of sulfoaluminates.

After the first contact between cement and water, the calcium concentration in the interstitial fluid of fresh cement pastes does not vary much with the water-to-cement mass ratio. In most cases, calcium ions are around saturation concentrations and favour the precipitation of portlandite. Conversely, the concentration of sulfate ions within cement suspensions varies depending on the water to cement mass ratio $(\mathrm{w} / \mathrm{c})$ along with the amount and solubility of calcium sulfate sources in the system [1].

In practice, the amount and type of sulfate calcium sources are generally chosen by the cement manufacturers by considering the $\mathrm{C}_{3} \mathrm{~A}$ and alkali sulfate contents present in the clinker. These can vary between gypsum $\left(\mathrm{CaSO}_{4} \cdot 2 \mathrm{H}_{2} \mathrm{O}\right)$, hemihydrate $\left(\mathrm{CaSO}_{4} \cdot 0.5 \mathrm{H}_{2} \mathrm{O}\right)$, anhydrite $\left(\mathrm{CaSO}_{4}\right)$ or mixtures of these sulfate sources. Even when faced with a pure gypsum-based system, through the grinding process, natural gypsum may dehydrate to hemihydrate depending on the mill ambient temperature. The final ground cement product results therefore into two different types of sulfate bearing materials with variant solubility.

This variability in sulfate sources dictates the sulfate concentration during the early hydration and consequently, chemical or physical interactions within the cement suspension [2]. Although the amount of hydrates formed during the first minute after the contact between water and cement is almost negligible, variations in sulfate concentrations have been found able to affect the macroscopic rheological behaviour of fresh cement pastes [3, 4]. Yamada in [5] demonstrated that the source of sulfate ions is of great significance for ettringite crystallisation. They found that the amount and the morphology of formed ettringite depend largely on the type and the quantity of calcium sulfate used. Ettringite is often considered as nucleating under the form of a hexagonal rod with six large elongated rectangular prism faces and two six sided end faces. Depending on the degree of super-saturation reached in the interstitial solution, which is itself driven by the sulfate source solubility, ettringite develops different morpholo-

¿ Open Access. (c) 2020 W. Mbasha et al., published by De Gruyter. (cc) BY 4.0 License 
gies that can vary from short hexagonal prisms to long needles [6-8].

To date, the effect of ettringite morphology on cement rheological parameters during the early hydration has not been thoroughly investigated. The main challenge in such investigations lies in the difficulty of assessing ettringite morphology at cement paste sample level. Relying solely on SEM analysis of cement samples containing only a few of these crystals should be considered only with care. In this work, packing measurements are laterally used as a fast and easy tool to confirm the SEM results. The w/c ratios of cement systems were kept constant along with the total amount of calcium sulfate. The calcium sulfate availability was changed by varying relative proportions of hemihydrate and natural gypsum powders as the source of calcium sulfate within the system.

\section{Materials and methods}

\subsection{Materials}

An ordinary Portland cement clinker was sampled during the production process of CEM I in accordance with ASTM C150 under stable kiln operations at a local cement plant using modern technology. This clinker was then crushed and ground without any calcium sulfate bearing material and was considered as the reference pure clinker. Two types of calcium sulfate materials, namely natural gypsum (NG) and hemihydrate ( $\mathrm{HH})$, were also considered. The chemical composition of the cement clinker and calcium sulfate materials as determined by XRF spectroscopy are presented in Table 1. The difference between these two cal-

Table 1: Chemical and phase composition of clinker and calcium sulfate

\begin{tabular}{cccc}
\hline Compounds & Clinker & Gypsum & Hemihydrate \\
\hline $\mathrm{SiO}_{2}$ & 23.2 & 0.81 & 1.01 \\
$\mathrm{Al}_{2} \mathrm{O}_{3}$ & 5.96 & 0.03 & 0.10 \\
$\mathrm{Fe}_{2} \mathrm{O}_{3}$ & 3.61 & 0.00 & 0.00 \\
$\mathrm{Mn}_{2} \mathrm{O}_{3}$ & 0.38 & 0.08 & 0.08 \\
$\mathrm{TiO}_{2}$ & 0.27 & 0.01 & 0.02 \\
$\mathrm{CaO}$ & 62.38 & $\mathbf{4 2 . 0 8}$ & $\mathbf{4 0 . 6 3}$ \\
$\mathrm{MgO}$ & 1.68 & 0.46 & 0.56 \\
$\mathrm{P}_{2} \mathrm{O}_{5}$ & 0.09 & 0.05 & 0.05 \\
$\mathrm{Cl}$ & 0.02 & - & - \\
$\mathrm{SO}_{3}$ & 0.81 & $\mathbf{4 4 . 7 1}$ & $\mathbf{5 2 . 4 3}$ \\
$\mathrm{K}_{2} \mathrm{O}$ & 0.45 & 0.03 & 0.03 \\
$\mathrm{Na}_{2} \mathrm{O}$ & 0.07 & - & - \\
\hline
\end{tabular}

cium bearing materials can mainly be depicted from their $\mathrm{CaO}$ and $\mathrm{SO}_{3}$ contents.

\subsubsection{Cement sample and characterisation}

Cement samples were obtained by adding directly to the clinker $4 \%$ of calcium sulfate from a mixture of natural gypsum and hemihydrate in different proportions. The prepared raw materials were homogenised in a ball mill bottle for a few hours as done in [9]. Cement samples were characterised by assessing their particle size distribution using a laser particle analyser (Malvern Mastersizer 2000 Malvern Instruments, UK) under constant agitation at $2000 \mathrm{rpm}$ in isopropanol as solvent. The equipment has the capacity to detect particles within the range of $0.02-2000 \mu \mathrm{m}$.

Particle size distributions of cement mixes are given in Table 2. It can be seen that the addition of $4 \%$ calcium sulfate bearing materials to the clinker does not have much effect on the specific surface area of the cement systems. The characteristic sizes such as $d_{10}, d_{50}$ and $d_{90}$ of all the cement mixes also remain similar to the original clinker size.

The total sulfate content of raw materials was estimated using a Leco and are presented in Table 3. The nomenclature $\mathrm{NG} / \mathrm{HH}$ means that the cement is made up of NG percent natural gypsum fraction and $\mathrm{HH}$ percent hemihydrate fraction within the gypsum cement phase set at $4 \%$ total mass concentration. It can be noted that, such procedure results in an effective sulfate content decrease of around $10 \%$ when totally substituting natural gypsum by hemihydrate within the cement system.

Table 2: Particle sizes distribution of the clinker and cement with different mass proportions of NG and HH for a $4 \%$ total mass of calcium sulfate within the system

\begin{tabular}{lcccc}
\hline $\begin{array}{l}\text { Sample ID/ } \\
\text { characteristic size }\end{array}$ & $\mathbf{d}_{\mathbf{1 0}}$ & $\mathbf{d}_{\mathbf{5 0}}$ & $\mathbf{d}_{\mathbf{9 0}}$ & $\begin{array}{c}\text { SSA } \\
{\left[\mathbf{c m}^{\mathbf{2}} / \mathbf{g}\right]}\end{array}$ \\
\hline Clinker & 5.96 & 16.4 & 36.1 & 3,840 \\
$\mathrm{NG}$ & 1.95 & 7.96 & 23.7 & 13,600 \\
$\mathrm{HH}$ & 1.82 & 8.69 & 42.9 & 13,700 \\
$100 / 0$ & 4.86 & 15.8 & 35.2 & 4,220 \\
$70 / 30$ & 5.35 & 16.2 & 36.5 & 3,990 \\
$60 / 40$ & 5.80 & 16.3 & 36.4 & 3,880 \\
$50 / 50$ & 5.32 & 16.1 & 36.2 & 3,990 \\
$40 / 60$ & 5.19 & 16.4 & 37.2 & 4,000 \\
$0 / 100$ & 4.85 & 15.9 & 35.1 & 4,230 \\
\hline
\end{tabular}


Table 3: Total $\mathrm{SO}_{3}$ concentration within the clinker with $4 \%$ calcium sulfate provided by a mixture of NG and $\mathrm{HH}$ in different proportions

\begin{tabular}{lcccccc}
\hline $\mathrm{NG} / \mathrm{HH}$ concentration & $0 / 100$ & $40 / 60$ & $50 / 50$ & $60 / 40$ & $70 / 30$ & $100 / 0$ \\
\hline $\mathrm{SO}_{3}[\%]$ & 2.89 & 2.56 & 2.97 & 2.46 & 2.69 & 2.38 \\
Mole of sulfate/g of cement & 0.69 & 0.63 & 0.62 & 0.61 & 0.59 & 0.55 \\
\hline
\end{tabular}

It is worth noting that, in most commercial cements, the total amount of $\mathrm{SO}_{3}$ is advised to be between $2.5-3 \%$ at a calcium sulfate content of not more than $5 \%$ [10-12].

\subsubsection{Cement paste preparation}

Unless specified, a constant w/c ratio of 0.4 is used throughout this experimental study. Cement paste is batched with a laboratory Hobart mixer using distilled water at a constant temperature of $23 \pm 1{ }^{\circ} \mathrm{C}$. Water is first in troduced into the mixer and the cement is gradually added over a period of $2 \mathrm{~min}$. After a resting time of about $2 \mathrm{~min}$, the mixing resumes for a further total mixing time of about $10 \mathrm{~min}$. A portion of the fresh cement is then collected for rheological measurements and stored in a water bath at $23 \pm 1^{\circ} \mathrm{C}$ for 15 minutes prior to the test. This curing time was suggested to allow for the precipitation of at least one third of the total ettringite amount [13]. The other portions are cast in small plastic containers and kept for an hour in a curing room at room temperature for TG-DSC and SEMSE analysis. The hydration reaction of the one hour hydrated cement paste is later stopped by immersing it in isopropanol for 24 hours. The solvent is successively changed after 1 hour and 15 hours thereafter, washed with diethyl ether as suggested in [14]. The sample is then collected and further stored for 1 day under vacuum drying at room temperature to remove the remaining isopropanol.

\section{Methods}

\subsection{Rheometry}

Rheological experiments were carried out on a stresscontrolled Anton Paar MCR 51 rheometer with a $50 \mathrm{~mm}$ diameter roughened parallel plate geometry and a set gap of $0.6 \mathrm{~mm}$. This rheometer is fitted with a circulating water bath keeping the sample isothermally at $23^{\circ} \mathrm{C}$ during all rheological measurements. The pre-shearing was done at $100 \mathrm{~s}^{-1}$ for $30 \mathrm{~s}$ to ensure a homogeneous distribution of the sample within the rheometer shear gap as described in [15]. Immediately after pre-shearing, the rheological measurements were performed.
The dynamic yield stress and the plastic viscosity were obtained from the flow curve by extrapolating the measured data of the down curve to zero shear rate using available rheological constitutive equations. Since the famous "Yield stress Myth" paper was published by Barnes and Walters in 1985 [16], much debate has raged on whether the yield stress is real or not. Much depends on the shear rate range over which data is measured. For most engineering applications the yield stress is a reality. The impact that rheological models have on accuracy and repeatability of rheological measurements have been reported in [17]. Although the Bingham model does not fit the experimental data well at low shear rates (due to the prevalence of a nonlinear relationship between applied shear rates and shear stresses), for cementitious materials, it is the most used model by virtue of its simplicity and the physical interpretation of its parameters [18]. For lower shear rates the modified Bingham or Herschel Buckley models are mostly used to adjust for this reality. Data collected within these regions however are not very reliable and difficult to reproduce as a result of the low torques applied [19]. Jayasree and Gettu [19] compared the Bingham (linear) and Herschel Buckley (polynomial) models for cement paste materials and found that both fit the experimental data well and provide comparable yield stress values. Compared to the Bingham model, the Herschel Buckley model provides higher yield stress values in case of shear thickening and lower values in shear thinning materials such as cement paste. They therefore recommend the Bingham model especially for parametric studies. The low shear rates that cementitious suspensions practically experience during casting, placing or pumping are within the applicable range of stresses that are well fitted by the Bingham model. In this investigation, experimental data were therefore fitted by the Bingham model. The measuring cycle time was $300 \mathrm{~s}$ with a shear rate ramp of $0-100 \mathrm{~s}^{-1}$ for both up and down curves.

\subsubsection{Thermogravimetric analysis}

A TGA/DSC Nersch STA model was used for the thermal gravimetric analysis. This is a simultaneous thermal analyser equipped with a data acquisition system using Ner- 
sch software. This allows the instrument to carry out at the same time the thermal gravimetric analysis and the differential scanning calorimetry of the sample. The base line was set by using an aluminium crucible pan. The nitrogen $\left(\mathrm{N}_{2}\right)$ flux was used as the flowing gas at $50 \mathrm{ml} / \mathrm{min}$. The heating started at $23^{\circ} \mathrm{C}$ at a rate of $5 \mathrm{~K} / \mathrm{min}$ in order to allow the equipment to stabilise beforehand and to eliminate all possible defects due to the setting up of the instrument. The reference sample was air and the experimental heating temperature ranged from $24-1000^{\circ} \mathrm{C}$. The hydrate phases were quantified by calculating the mass loss within the specified range of temperatures as described in [20,21].

\subsubsection{Packing density measurements}

The morphology of ettringite was assessed by measuring the paste packing properties after contact with water. Pastes with different proportions of natural gypsum and hemihydrate were prepared with distilled water at a w/c ratio of 0.5 . After mixing, the paste was kept in a sealed container at room temperature for 1 hour allowing for sufficient precipitations of early hydrate products (ettringite). Thereafter, the paste was remixed, and $1.5 \mathrm{~mL}$ was collected, placed in a specified tube and transferred to the centrifuge equipment. The centrifuge was done at 5000 rpm (4193 g) for 10 minutes. The liquid on top was removed and weighed on an electronic balance. This was preferred to measuring the height of compacted cement due to the fact that, after centrifuging, the paste was not evenly spread around the circumference of the tube. The solid volume fraction of the consolidated suspension, which corresponds to the packing density of the suspension under these experimental conditions (duration and acceleration), was then computed.

\subsubsection{Scanning electron microscopy}

The microstructure of the hydrated cement systems was analyzed by scanning electron microscopy (SEM-SE) and their atomic ratio was assessed by energy dispersive X-ray spectroscopy (EDS) analysis. The samples were prepared as described in section 2.1.2 and further treated for SEM measurements. Samples were impregnated in LR White epoxy-based resin overnight and polished using $100 \mathrm{~nm}$ fine diamond lapping paper to create a flat surface required for quantitative EDS analyses. The samples were thereafter coated with a thin carbon film $(<5 \mathrm{~nm})$. A Zeiss Auriga field emission gun scanning electron microscope (FEG-SEM) operated at $15 \mathrm{kV}$ for imaging and $20 \mathrm{kV}$ for EDS analyses was used during analysis; all EDS analyses were completed using an Oxford Instruments X-Max solid-state silicon drift detector.

\section{Experimental results}

\subsection{Flow properties}

The yield stress and viscosity values of the cements were estimated from typical flow curves as shown in Figure 1. It was noticed that, the characterisation of all down curves of hysteresis loops on flow curves could be fitted with the Bingham model. However, cement systems with only hemihydrate presented higher Bingham parameters than those with pure natural gypsum. These parameters seemed to change with respect to the replacement of natural gypsum by hemihydrate in cement systems with mix proportions of these two calcium-sulfate bearing materials. The effect of the presence of hemihydrate within the gypsum on the flow behaviour of cement paste can be seen by establishing a relationship between these obtained rheological parameters and the actual concentration of hemihydrate as illustrated in Figure 2.

It can be observed from Figure 2 that, the presence of hemihydrate above a critical fraction around $60 \%$, results in an increase in both yield stress and plastic viscosity of the cement pastes. In particular, below 50\% hemihydrate fraction, yield stress values are similar to those of cement with only natural gypsum. Above this critical concentration, cement paste yield stress values and plastic viscosity significantly increase.

\subsection{Effect on ettringite formation}

The quantity of ettringite and portlandite were estimated based on the results from thermogravimetric and differential scanning calorimetry (TG-DSC) as presented in Figure 3. These characteristic TG-DSC curves clearly show appreciable mass loss occurring between the ranges of 100$140^{\circ} \mathrm{C}$ for all cement pastes after one hour of hydration. This is mainly attributed to the dehydration of ettringite. Modest mass losses are also depicted in a temperature range of $170-200^{\circ} \mathrm{C}$ in all cements. This loss is mainly attributed to the dehydration of AFm (calcium hydroaluminate or monosulfate) phase [22]. Further mass losses are noticed within the ranges of $350-530^{\circ} \mathrm{C}$ corresponding to the de-hydroxylation of portlandite. The decarbonation of 

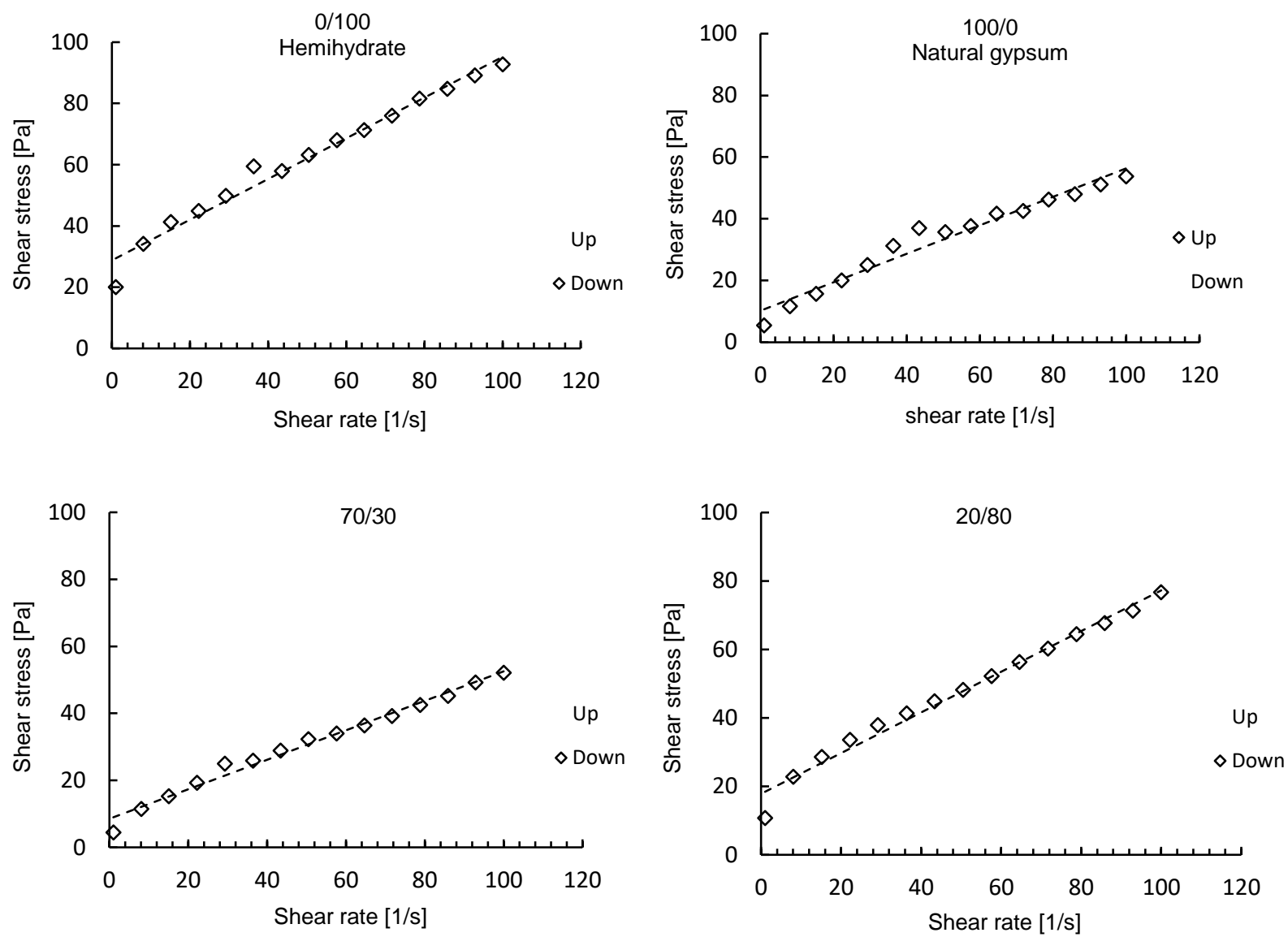

Figure 1: Typical flow curves of cement pastes with different proportions of natural gypsum and hemihydrate within gypsum cement phase.
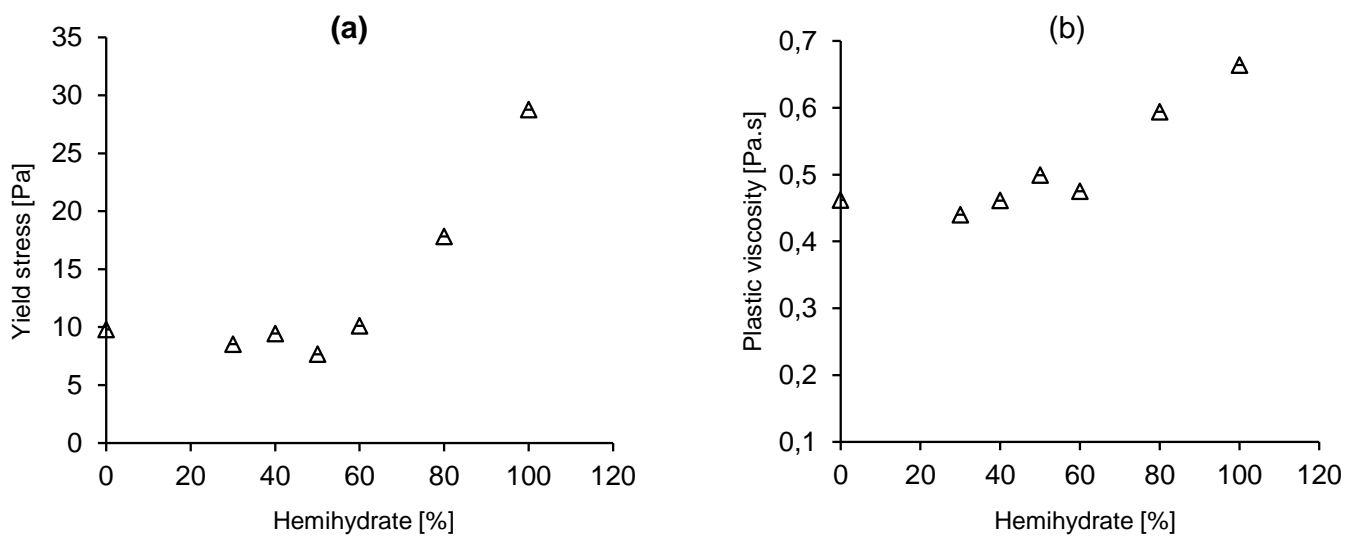

Figure 2: Effect of hemihydrate fraction among the total calcium-sulfate bearing materials on (a) yield stress and (b) plastic viscosity.

carbonated phases are depicted between a temperature range of $600-900^{\circ} \mathrm{C}$.

Figure 4 shows the quantity of ettringite and portlandite estimated within specified temperature ranges as described in [23-25]. After 1 hour of hydration, it can be observed that, increasing the hemihydrate fraction within the cement system did not result in any major increase in the amount of formed hydrate products. In all systems the average amount of ettringite formed was $4.6 \%$ and portlandite $1.8 \%$. 

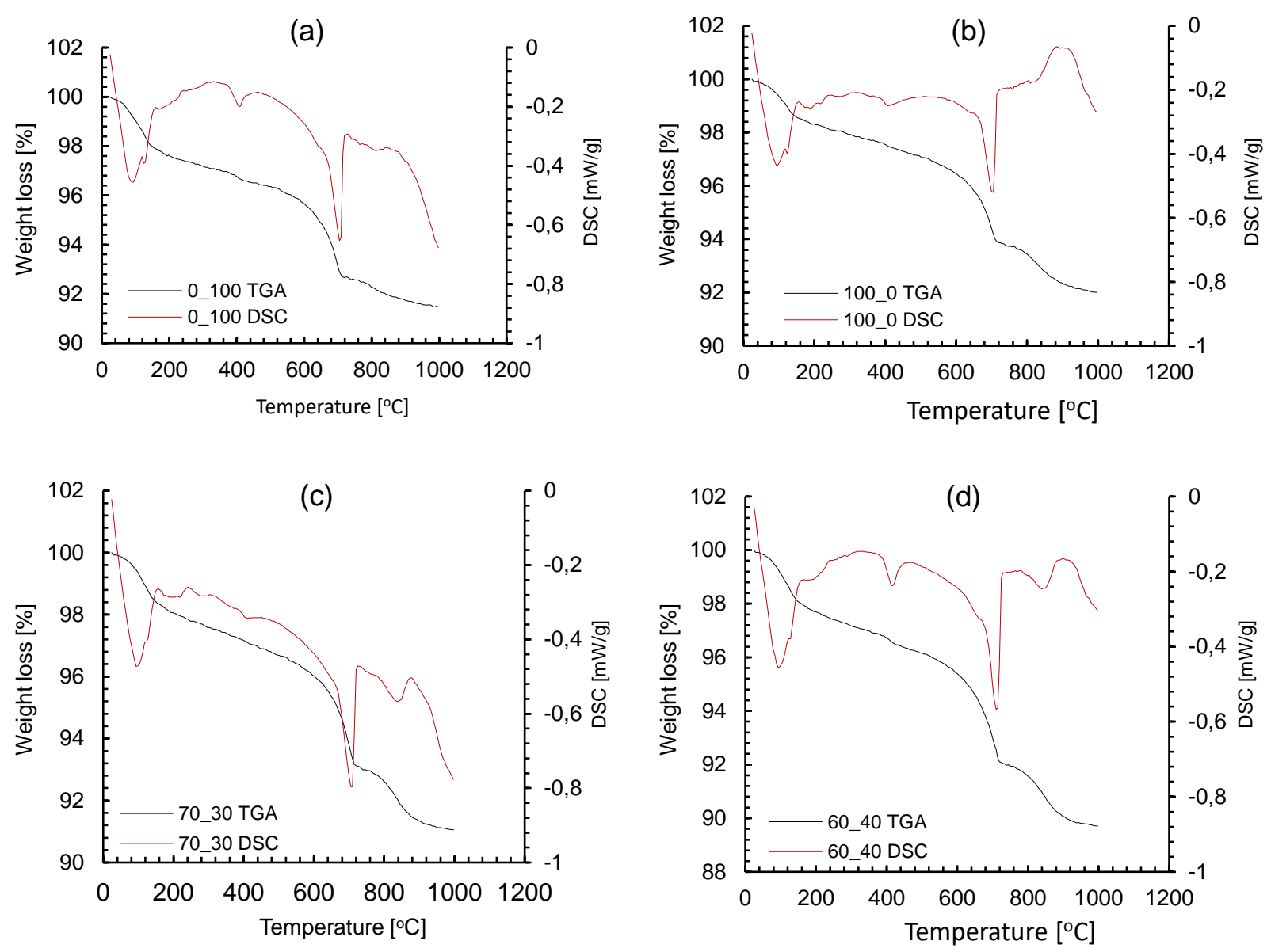

Figure 3: Typical thermogravimetric analysis and differential scanning calorimetry of one hour hydrated cement pastes with different proportions of natural gypsum and hemihydrate within the gypsum phase set at $4 \%$. (a): Cement with hemihydrate $0 / 100$; (b): cement with natural gypsum 100/0; (c): cement with $70 / 30$ proportion; (d): cement with 60/40 proportion.
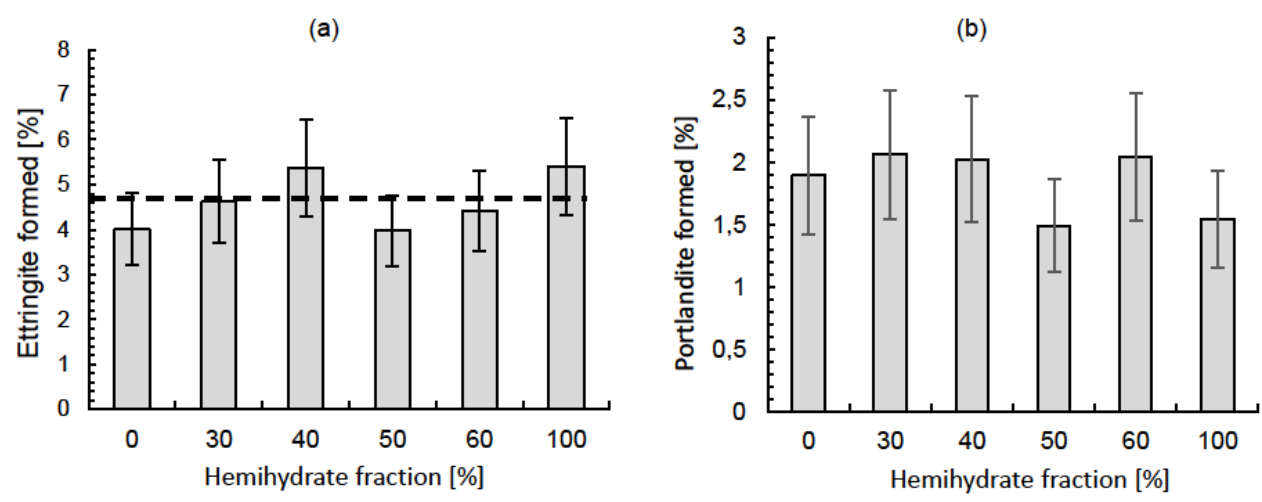

Figure 4: Amount of hydrate products precipitated after 1 hour of cement hydration with different fractions of hemihydrate within the sulfate-carriers; (a): Ettringite and (b): Portlandite. The dashed line is the average of all measurements.

\subsection{Effect on packing density}

Figure 5 presents the packing densities measured at an accuracy estimated around $0.2 \%$ for all hemihydrate concentrations within the cement systems. It can be seen that, the packing density of the suspension stays almost the same up to the $50 \%$ hemihydrate fraction and thereafter decreases with the increase in hemihydrate concentration. This implies that the paste has higher packing properties when natural gypsum was solely used or mixed with hemi- 
hydrate up to $50 \%$. Beyond this concentration, the packing density decreases from $44.4 \%$ to $43.4 \%$ indicating a less compacted paste.

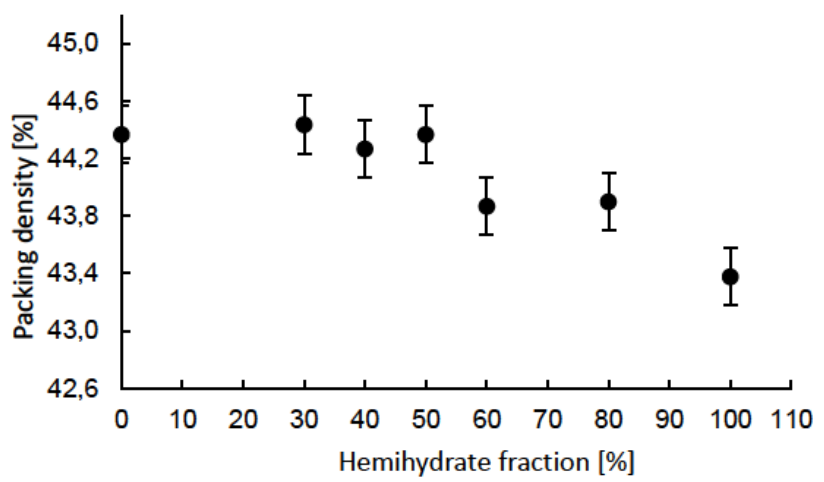

Figure 5: Effect of hemihydrate fraction within cement gypsum phase on cement paste packing.

\section{Discussion}

Results of this research suggest that, rheological properties of cement paste are more influenced by the morphology than the amount of precipitated ettringite during the early hydration. Evidently, this morphology depends on both the presence and content of hemihydrate within the cement paste.

\section{Morphology of ettringite and sulfate sources}

Ettringite is the main stable hydrate product precipitating from the interaction of aluminate and calcium sulfate phases during the early stage of ordinary Portland cement hydration. This hydrate precipitates until sulfate depletion $[26,27]$. Previous research has shown that the type and content of sulfate used as set regulator can also affect the chemical structure of ettringite thus altering the performances of cement paste [4, 28-31].

On the one hand, a significant amount of sulfate ions within the suspension results in a large amount of ettringite formation that, in turn, may affect hydration kinetics and cause microstructure expansion [32, 33]. On the other hand, Evju and Hansen [34] found that the morphology of ettringite formation depends on the source of the sulfate used. They observed radial crystal ettringite in the presence of $\beta$-hemihydrate and, when mixed with another source of sulfate, its crystal growth was altered. Yamada and $\mathrm{Xu}[4,35]$ have also shown and reported the signifi- cance of these sulfate-source parameters on ettringite crystallisation. It is thus understood from literature that the morphology of formed ettringite largely depends on the type and content of calcium sulfate used.

\section{Rheology and its driving parameters}

Flatt and Bowen [36] have shown that the yield stress of traditional cement paste depends on inter-particle attractive forces, solid volume fraction, particle size and maximum packing density. On the one hand, the maximum attractive force between cement particles is assumed to come from non-retarded Van der Waals forces. On the other hand, cohesion forces which originate from C-S-H nucleation can considerably increase cement yield stress values at rest, due to the bridging that $\mathrm{C}-\mathrm{S}$-H provides between cement particles [37].

It was shown in $[38,39]$ that hydration of tricalcium aluminate along with sulfate ions that are not consumed by ettringite formation may affect the growth of these C$\mathrm{S}-\mathrm{H}$ and potentially cohesion forces they induce. However as soon as the mixing power is sufficient to break the bonds which originates from C-S-H nucleation, the effect of the corresponding cohesion forces on the yield stress becomes negligible [8]. In this work, rheological measurements were made immediately after the samples were presheared at a high shear rate and the attractive forces at the origin of the dynamic yield stress are therefore assumed to be independent of hydrates formation.

For non-Brownian and non-colloidal particles, viscosity increases with solid volume fraction and diverges when the maximum packing density is approached [40]. For colloidal flocculated suspensions like cement paste, it was shown that their viscosity mostly depends on the surfaceto-surface separating distance between cement grains imposed by attractive Van der Waals forces [41] and the potential presence of adsorbed plasticisers.

Within the above framework, both yield stress and viscosity depends on the ratio between solid volume fraction and maximum packing fraction. As the volume variations induced by hydration at such early ages was negligible, variations in maximum packing fraction are expected to have a strong influence on rheological parameters. As maximum packing fraction mostly depends on particle morphology and polydispersity, modifications in the cement paste rheological behaviour are expected if the sulfate source affects the morphology of hydrate products changing therefore the maximum packing fraction of the system. 

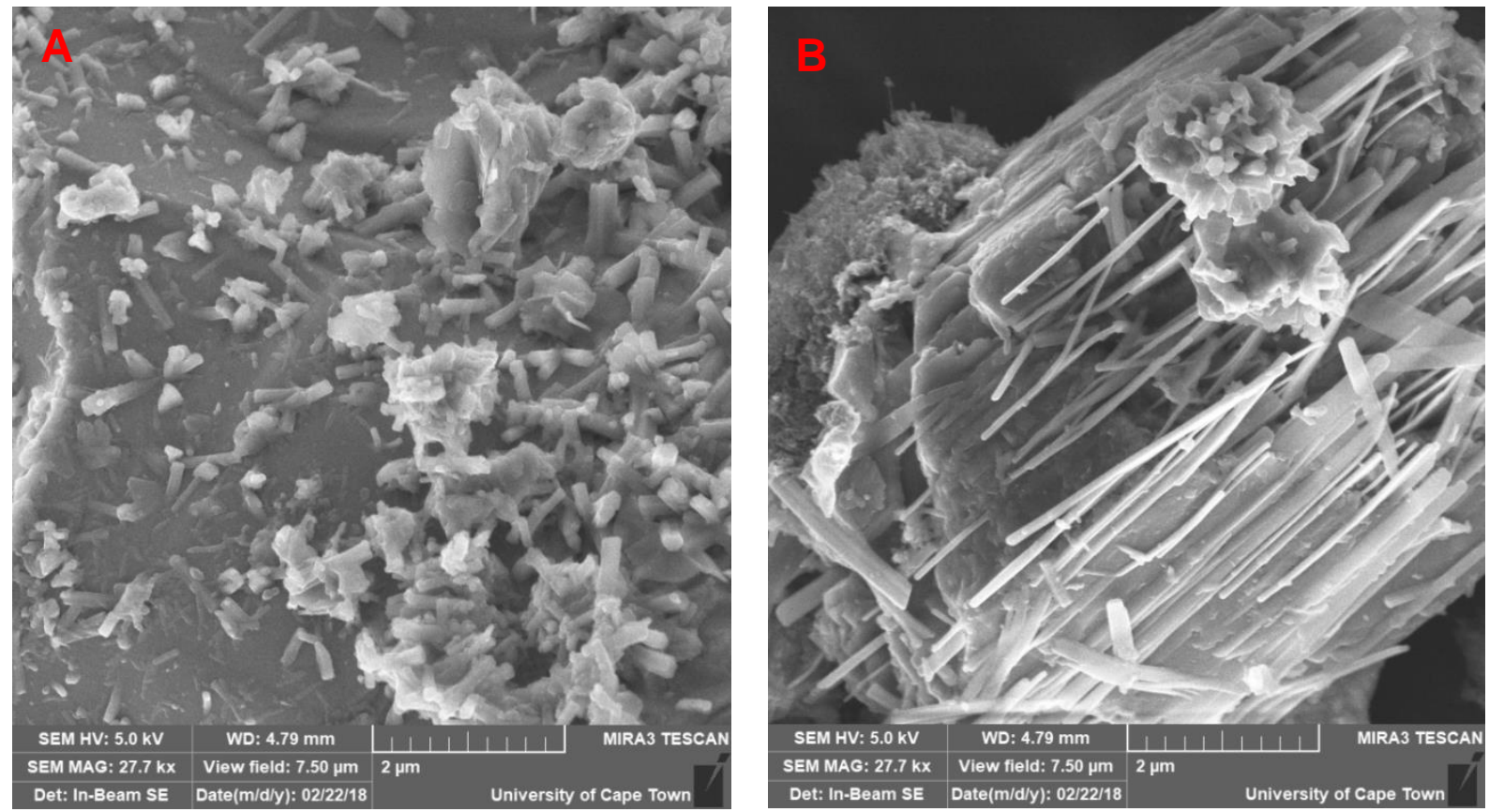

Figure 6: SEM-SE images of cement surface hydrated for 1 hour and prepared with (A) natural gypsum and (B) hemihydrate.

\section{Total ettringite formed in the system}

In the tens of minutes following the contact of cement and water, calcium, sulfate and hydroxyl ions are released into the suspension [42-45]. These ions are consumed for the formation of hydrate products, mainly ettringite and portlandite [46]. The amount of these two hydrates after one hour of hydration is shown in Figure 4. We note that the variations of these early hydrate products were respectively around $0.5 \%$ and $1 \%$ and were of the order of the uncertainty of our measurements.

Assuming the density of cement, ettringite and portlandite to be $3.15 \mathrm{~g} / \mathrm{cm}^{3}, 1.8 \mathrm{~g} / \mathrm{cm}^{3}$ and $2.4 \mathrm{~g} / \mathrm{cm}^{3}$ respectively, the variations in solid volume fraction due to those of ettringite and portlandite can therefore be estimated to be $1 \%$ and $0.5 \%$ respectively. At such variations, the solid volume fraction of ettringite could slightly affect the rheological behaviour of the suspension. There was however no correlation found between the solid volume fractions and the observed rheological parameters of the corresponding cement paste. These observations are in agreement with those reported by [47] who also found no correlation between variations in the amount of ettringite formed and the rheological properties of cement pastes.

\section{Ettringite morphology and packing fractions}

Maximum packing density measurements in Figure 5 suggest that the increase of the hemihydrate fraction alters the packing of the suspension. Considering that the particle size distribution of cement particles remains almost unchanged during the first hour of hydration, the only factor that might affect the maximum packing fraction of the suspension is the shape of the particles. A variation in the morphology of ettringite could explain the modification of the packing properties. Figure 6 (A) and (B) show the SEM-SE morphology images of ettringite at the surface of cement particles hydrated during 1 hour in the presence of natural gypsum and hemihydrate. Elongated rod-like ettringite hydrates are observed at the surface of cement with hemihydrate while short rod-like ettringite hydrates are seen in the presence of natural gypsum. A similar phenomena pertaining to ettringite morphology changes resulting from aluminate and calcium sulfate interactions in different cement systems have also been reported by $[4,48]$.

We note from Figure 6 that the presence of these elongated hydrates at the surface of cement particles affect the regularity of their shape. Previous research has shown that particles with regular shapes and flat surfaces locally arrange themselves better than those with irregular shapes [49-51]. When a long rod is inserted into a packing of particles and if it is not small enough to occupy the space between particles, the overall packing density of the system decreases accordingly. We suggest therefore that the decrease in packing density with the increase in hemihydrate fraction within the cement system can be attributed to the elongated shape of ettringite formed. 
Consequently, it can tentatively be assumed that, at concentrations below 50\% hemihydrate, spherical or stubby rod ettringite prevails within the suspension and above this concentration elongated ettringite dominates. Interestingly, the trend observed in Figure 6 converges with that of rheological parameters as illustrated in Figure 2.

Considering this, rheological properties of fresh cement paste seem to be controlled more by the morphology than the available amount of ettringite triggered by the presence of a high concentration of hemihydrate within the suspension. These observations would thus explain the decrease of the packing density, which results in the increase of both yield stress and viscosity as the hemihydrate fraction increases.

\section{Conclusions}

The results obtained in this research show that more than the amount of ettringite precipitated, ettringite morphology plays a major role in controlling the packing properties and consequently the yield stress and plastic viscosity of fresh cement pastes. This morphology strongly depends on the change in sulfate availability depending, for instance, on the presence of the hemihydrate fraction within the gypsum phase.

This leads to a large increase in yield stress values and a variation in plastic viscosity value of approximately $50 \%$.

In practice, it is difficult to control the availability or sulfate consumption within cement suspensions for concrete rheological purposes. This makes it challenging to directly implement this approach as a rheology control tool. However, considering what has been shown in this research, it is suggested that practical and effective ways for changing the ettringite morphology be investigated by incorporating intercalating polymers.

Acknowledgement: PPC Ltd. is acknowledged for sponsoring the research and for making available the chemical and physical properties of the cements tested.

\section{References}

[1] Platel D., Impact de l'architecture macromoléculaire des polymères sur les propriétés physico-chimiques des coulis de ciment, Université Pierre et Marie Curie, 2005.

[2] Salvador R. P., Cavalaro S. H. P., Cincotto M. A., and Figueiredo A. D. de, Parameters controlling early age hydration of cement pastes containing accelerators for sprayed concrete, Cem. Concr. Res. 2016, 89, 230-248.

[3] Flatt R. J., Zimmermann J., Hampel C., Kurz C., Schober I., Frunz L., et al., The Role of Adsorption Energy in the SulfatePolycarboxylate Competition, Int. Concr. Abstr. Portal, 2009, 262, 153-164.

[4] Yamada K., Ogawa S., and Hanehara S., Controlling of the adsorption and dispersing force of polycarboxylate-type superplasticizer by sulfate ion concentration in aqueous phase, Cem. Concr. Res., 2001, 31, 375-383.

[5] Prince W., Espagne M., and Aiẗcin P.C., Ettringite formation: A crucial step in cement superplasticizer compatibility, Cem. Concr. Res., 2003, 33, 635-641.

[6] Marchon D., and Flatt R. J., Chapter 8, in Mech. Hydration, Wodhead Publishing, New York, 2016, pp. 129-145.

[7] Mehta P., Morphology of calcium sulfoaluminate hydrates, J. Am. Ceram. Soc. 1969, 52, 521-522.

[8] Meredith P., and Donald M., Tricalcium aluminate hydration: Microstructural observations by in-situ electron microscopy, J. Mater. Sci., 2004, 39, 997-1005.

[9] Li X., Xu W., Wang, S., Tang M., and Shen X., Effect of $\mathrm{SO}_{3}$ and $\mathrm{MgO}$ on Portland cement clinker: Formation of clinker phases and alite polymorphism, Constr. Build. Mater. 2014, 58, 182-192.

[10] Ku H. H., Statistical concepts in metrology-with a postscript of statistical graphics, US Dept. of Commerce, National Bureau of Standards, 1988.

[11] Peray K. E., Cement Manufacturer's Handbook, Chemical Publishing Co.,Inc., New York, 1979.

[12] AENOR.UNE-EN 197-1 E. S., Cement - Part 1: Composition, Specifications and Conformity Criteria for Common Cements, 2011.

[13] Dalas F., Pourchet S., Rinaldi D., Nonat A., Sabio S., and Mosquet M., Modification of the rate of formation and surface area of ettringite by polycarboxylate ether superplasticizers during early C3A-CaSO4 hydration, Cem. Concr. Res., 2015, 69, 105-113.

[14] Scrivener K., Snellings R., and Lothenbach B., A Practical Guide to Microstructure Analysis of Cementitious Materials, CRC Press, New York, 2015.

[15] Mezger T. G., The rheology handbook: for users of rotational and oscillatory rheometers, Vincentz Network GmbH \& Co KG, 2006.

[16] Barnes H. A., and Walters K., The yield Stress Myth, Rheol. Acta 1985, 24, 323-326.

[17] Nehdi M., Rahman M.A., Estimating rheological properties of cement pastes using various rheological models for different test geometry, gap and surface friction, Cem. Concr. Res, 2004, 34, 1993-2007.

[18] Ke G., Zhang J., Xie S., Pei T., Rheological behaviour of calcium sulfoaluminate cement paste with supplementary cementitious materials, Constr. Build. Mater, 2020, 243, 118234.

[19] Jayasree C., Gettu R., Experimental study of the flow behaviour of superplasticized cement paste, Mater. Struct, 2008, 41, 15811593.

[20] Collier N. C., Sharp J. H., Milestone N. B., Hill J., and Godfrey I. H., The influence of water removal techniques on the composition and microstructure of hardened cement pastes, Cem. Concr. Res., 2008, 38, 737-744.

[21] Monteagudo S. M., Moragues A., Gálvez J. C., Casati M. J., and Reyes E., The degree of hydration assessment of blended cement pastes by differential thermal and thermogravimetric analysis. Morphological evolution of the solid phases, Thermochim. Acta, 2014, 592, 37-51. 
[22] Odler I., and Abdul-Maula S., Possibilities of quantitative determination of the AFt-(ettringite) and AFm-(monosulfate) phases in hydrated cement pastes, Cem. Concr. Res., 1984, 14, 133-141.

[23] Meller N., Kyritsis K., and Hall C., The hydrothermal decomposition of calcium monosulfoaluminate 14-hydrate to katoite hydrogarnet and $\beta$-anhydrite: An in-situ synchrotron X-ray diffraction study, J. Solid State Chem., 2009, 182, 2743-2747.

[24] Wang X., Pan Z., Shen X., and Liu W., Stability and decomposition mechanism of ettringite in presence of ammonium sulfate solution, Constr. Build. Mater., 2016, 124, 786-793.

[25] Zhang Q., and Ye G., Quantitative analysis of phase transition of heated Portland cement paste, J. Therm. Anal. Calorim., 2013, 112, 629-636.

[26] Danner T., Justnes H., Geiker M., and Lauten R. A., Early hydration of $\mathrm{C}_{3} \mathrm{~A}$-gypsum pastes with $\mathrm{Ca}$ - and Na-lignosulfonate, $\mathrm{Cem}$. Concr. Res., 2016, 79, 333-343.

[27] Evju C., and Hansen S., Expansive properties of ettringite in a mixture of calcium aluminate cement, Portland cement and $\beta$-calcium sulfate hemihydrate, Cem. Concr. Res., 2001, 31, 257261.

[28] Roller P. S., The Setting of Portland Cement, Ind. Eng. Chem., 1934, 26, 669-677.

[29] Shi C., Zhang G., He T., and Li Y., Effects of superplasticizers on the stability and morphology of ettringite, Constr. Build. Mater., 2016, 112, 261-266.

[30] Tzouvalas G., Rantis G., and Tsimas S., Alternative calciumsulfate-bearing materials as cement retarders: Part II. FGD gypsum, Cem. Concr. Res., 2004, 34, 2119-2125.

[31] Zhang H., Zhongsou L., and Tong D., Influence of the type of calcium sulfate on the strength and hydration of Portland cement under intial steam-curing condition, Cem. Concr. Res., 1996, 26, 1505-1511.

[32] Quennoz A., Hydration of $C_{3} A$ with Calcium Sulfate Alone and in the Presence of Calcium Silicate, doctoral thesis, Ecole Polytechnique Federale de Lausanne, 2011.

[33] Zhang S., Xu X., Memon S. A., Dong, Z., Li D., and Cui H., Effect of calcium sulfate type and dosage on properties of calcium aluminate cement-based self-leveling mortar, Constr. Build. Mater. 2018, 167, 253-262.

[34] Evju C., and Hansen S., The kinetics of ettringite formation and dilatation in a blended cement with $\beta$-hemihydrate and anhydrite as calcium sulfate, Cem. Concr. Res., 2005, 35, 2310-2321.

[35] Xu L., Wang P., and Zhang G., Formation of ettringite in Portland cement/calcium aluminate cement/calcium sulfate ternary system hydrates at lower temperatures, Constr. Build. Mater., 2012, 31, 347-352.
[36] Flatt R. J., and Bowen P., Yodel: A Yield Stress Model for Suspensions, J. Am. Ceram. Soc., 2006, 89, 1244-1256.

[37] Roussel N., Ovarlez G., Garrault S., and Brumaud C., The origins of thixotropy of fresh cement pastes, Cem. Concr. Res., 2012, 42, 148-157.

[38] Gauffinet-Garrault S., The rheology of cement during setting, Underst. Rheol. Concr., 2011, 96.

[39] Garrault-Gauffinet S., and Nonat A., Experimental investigation of calcium silicate hydrate (CSH) nucleation, J. Cryst. Growth, 1999, 200, 565-574.

[40] Krieger I. M., and Dougherty T. J., A mechanism for nonNewtonian flow in suspensions of rigid spheres, Trans. Soc. Rheol., 1959, 1957-1977, 3, 137-152.

[41] Hot J., Bessaies-Bey H., Brumaud C., Duc M., Castella C., and Roussel N., Adsorbing polymers and viscosity of cement pastes, Cem. Concr. Res., 2014, 63, 12-19.

[42] Chung D. D. L., Electrical conduction behaviour of cement-matrix composites, J. Mater. Eng. Perform., 2002, 11, 194-204.

[43] El-Enein Abo S. A., Kotkata M. F., Hanna G. B., Saad M., and Abd El Razek M. M., Electrical conductivity of concrete containing silica fume, Cem. Concr. Res., 1995, 25, 1615-1620.

[44] Heikal M., Morsy M. S., and Aiad I., Effect of polycarboxylate superplasticizer on hydration characteristics of cement pastes containing silica fume, Ceram. Silik., 2006, 50, 5.

[45] Tamás F. D., Farkas E., Vörös M., and Roy D. M., Low-frequency electrical conductivity of cement, clinker and clinker mineral pastes, Cem. Concr. Res., 1987, 17, 340-348.

[46] Nalet C., and Nonat A., Impacts of hexitols on the hydration of a tricalcium aluminate-calcium sulfate mixture, Cem. Concr. Res., 2016, 89, 177-186.

[47] Bonen D., and Sarkar S. L., The superplasticizer adsorption capacity of cement pastes, pore solution composition, and parameters affecting flow loss, Cem. Concr. Res., 1995, 25, 1423-1434.

[48] Pourchet S., Regnaud L., Perez J. P., and Nonat A., Early C3A hydration in the presence of different kinds of calcium sulfate, Cem. Concr. Res., 2009, 39, 989-996.

[49] Aste T., and Weaire D., The Pursuit of Perfect Packing, Institute of Physics, Bristol, 2000.

[50] Cumberland D., and Crawford R., The packing of particles, Elsevier, Amsterdam, 1987.

[51] German R. M., Particle packing characteristics, Metal Powder Industry Federation, Princeton, 1989. 